\title{
FEM Analysis of Nb-Sn Rutherford-type Cables
}

\author{
Emanuela Barzi, Giuseppe Gallo, and Paolo Neri
}

\begin{abstract}
An important part of superconducting accelerator magnet work is the conductor. To produce magnetic fields larger than $10 \mathrm{~T}$, brittle conductors are typically used. For instance, for $\mathrm{Nb}_{3} \mathrm{Sn}$ the original round wire, in the form of a composite of Copper, Niobium and Tin, is assembled into a so-called Rutherford-type cable, which is used to wind the magnet. The magnet is then subjected to a high temperature heat treatment to produce the chemical reactions that make the material superconducting. At this stage the superconductor is brittle and its superconducting properties sensitive to strain. This work is based on the development of a 2D finite element model, which simulates the mechanical behavior of Rutherford-type cable before heat treatment. The model was applied to a number of different cable architectures. To validate a critical criterion adopted into the single $\mathrm{Nb}$-Sn wire analysis, the results of the model were compared with those measured experimentally on cable cross sections.
\end{abstract}

Index Terms- $\mathrm{Nb}_{3} \mathrm{Sn}$ wires, Rutherford-type cables, Restacked-Rod Process, Finite Element Model analysis, Principal strain, Plastic work

\section{INTRODUCTION}

$\mathrm{W}$ ITH progress and the continuous interest in A15 materials for superconducting magnets, a number of finite element models of deformed wires and cables were developed both in the High Energy Physics [1]-[5] and the Fusion $[6,7]$ communities. The analysis of $\mathrm{Nb}_{3} \mathrm{Sn}$ Rutherfordtype cables herein described builds upon a detailed model [4] of Restacked-Rod Processed (RRP) wires manufactured by Oxford Superconducting Technology [8, 9]. A large body of experimental data on flat-rolled RRP strands with 102, 108 and $114 \mathrm{Nb}$-Sn subelements was used in [4] to perform an accurate analysis of the failure mechanisms in the Nb-Sn composite. A study on 214 strand samples of RRP wire with $108 \mathrm{Nb}-\mathrm{Sn}$ subelements deformed between $10 \%$ and $30 \%$ showed that breakage in this wire starts at $26 \%$ deformation. Subelement deformations were measured on wire cross sections and compared with those obtained from the model's displacements. The good correlation between model and data allowed identifying a critical criterion for RRP wires. For the $\mathrm{Nb}-\mathrm{Sn}$ bundles not to merge and start breaking, the principal traction strain in the Cu should not exceed $0.48 \pm 0.10$.

A reliable cable model would allow evaluating for each contemplated cable geometry what is the maximum strand deformation during fabrication, what are the most critical

Manuscript received September 12, 2011.

This work was supported by the U.S. Department of Energy.

All authors are with the Fermi National Accelerator Laboratory (Fermilab), P.O. Box 500, Batavia, IL 60510 USA (phone: 630-840-3446; fax: 630-8403369; e-mail: barzi@fnal.gov). strand locations, and ideally predicting damage whenever the failure mechanisms of a specific strand technology are known. However, detailed models are typically too heavy to model entire Rutherford-type cables. Therefore, at first the cable was modeled by simplifying the strand geometry. The central hexagonal area which included the $\mathrm{Nb}-\mathrm{Sn}-\mathrm{Cu}$ hexagonal bundles was replaced by a homogeneous region with average properties weighted on the area percentage of each component. Such percentages were then corrected in order to obtain in the simplified strand model the same displacements as in the original detailed model. For the first rectangular forming stage of the cable, to reproduce the compaction effect of the top and bottom rollers and of the side rollers, vertical and lateral displacement values were imposed. To determine how to distribute the total lateral displacement (whether to apply it all on an edge of the cable or equally distribute it between left and right edges), a number of actual cables were modeled and the strands displacements from the model compared with those experimentally measured on the actual cable cross sections. The second keystoning stage of cable fabrication was modeled by imposing an additional vertical displacement varying linearly on the strands. A study was performed on the effect of the number of strands $N$ in the cable on maximum strand deformation. In order to identify local strain and critical locations inside the composite strands in the cable, the most critical strands in the cable cross section were modeled in detail by using surface displacements obtained through the simplified cable model. Thanks to this detailing, the effect of width compaction and of other cable parameters on maximum strain was studied. In the following, the model, the comparison with the data, the identification of critical locations, and the various sensitivity analyses are described.

\section{NB-SN RUTHERFORD-TYPE CABLE ANALYSIS}

\section{A. Detailed and Simplified FEM Strand Model}

Table I shows the wire parameters of the RRP strands used in this study. Fig. 1 shows a schematic of the detailed (left) [4] and simplified (right) $\mathrm{Nb}-\mathrm{Cu}-\mathrm{Sn}$ composite wire.

TABLE I WIRE PARAMETERS

\begin{tabular}{lcc}
\hline \hline Strand ID & A & B \\
\hline Stack design & RRP & RRP \\
Configuration & $108 / 127$ & $108 / 127$ \\
Diameter, mm & 1.0 & 0.7 \\
$\mathrm{~J}_{\mathrm{c}}(4.2 \mathrm{~K}, 12 \mathrm{~T}), \mathrm{A}$ & $>2600$ & $>2600$ \\
Cu fraction, \% & 54 & 54 \\
Max. subelement size, $\mu \mathrm{m}$ & 75 & 50 \\
Twist pitch, mm & 12 & 12 \\
\hline \hline
\end{tabular}



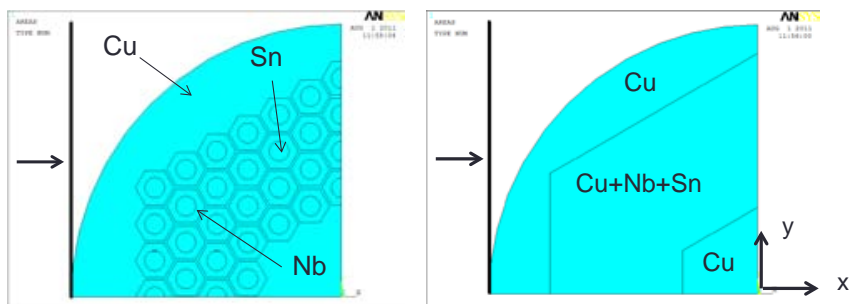

Fig. 1. Detailed (left) [4], and simplified (right) strand model.

The material properties used in the 2D ANSYS model [1113] are shown in Table II. The hypotheses used were of plain strain and isotropic behavior. The loads were provided as displacement of a rigid contact element (Target-169) applied gradually on the external surface of the wires. A flexible contact element was used on the wires surface (Contact-172).

TABLE II MATERIAL ELASTIC AND PlastiC PROPERTIES

\begin{tabular}{ccccl}
\hline \hline Element & $\begin{array}{c}\text { Young } \\
\text { Modulus, GPa }\end{array}$ & $\begin{array}{c}\text { Poisson } \\
\text { Modulus }\end{array}$ & $\begin{array}{c}\text { Tangent } \\
\text { Modulus, GPa }\end{array}$ & $\begin{array}{c}\text { Yield tensile } \\
\text { Strength, MPa }\end{array}$ \\
\hline $\mathrm{Cu}$ & 110 & 0.364 & 1.1 & 280 (cold worked) \\
$\mathrm{Nb}$ & 103 & 0.380 & 1.03 & 420 (small size rod) \\
$\mathrm{Sn}$ & 41.4 & 0.330 & 0.41 & $20(2.6 \mathrm{Bi}$ wt $\%)$ \\
\hline \hline
\end{tabular}

TABLE III ELEMENT COMPOSITION

\begin{tabular}{ccccc}
\hline \hline \multirow{2}{*}{ Strand ID } & \multicolumn{2}{c}{ A } & \multicolumn{2}{c}{ B } \\
& Original & Modified & Original & Modified \\
\hline $\mathrm{Cu} \%$ & 0.206 & 0.156 & 0.234 & 0.134 \\
$\mathrm{Nb} \%$ & 0.544 & 0.414 & 0.497 & 0.397 \\
$\mathrm{Sn} \%$ & 0.250 & 0.430 & 0.269 & 0.469 \\
\hline \hline
\end{tabular}

Table III specifies how element percentages were modified in the hexagonal $\mathrm{Nb}-\mathrm{Sn}-\mathrm{Cu}$ homogeneous region to obtain in the simplified strand model the same displacements as in the original detailed model. For strand A, the difference on the maximum y-component of the displacement ranged between $0.49 \%$ and $1.47 \%$ for wire deformation between $18 \%$ and $30 \%$. For strand $\mathrm{B}$, the difference on the maximum $\mathrm{y}$ component of the displacement ranged between $0.82 \%$ and $2.34 \%$ for wire deformation between $18 \%$ and $30 \%$.

Figs. 2 and 3 compare the y-component of displacement and the plastic work as obtained with the detailed wire model (left figures) and the simplified one (right figures). As can be seen, the simplified model reproduces displacements very well, but does not represent local effects in detail.

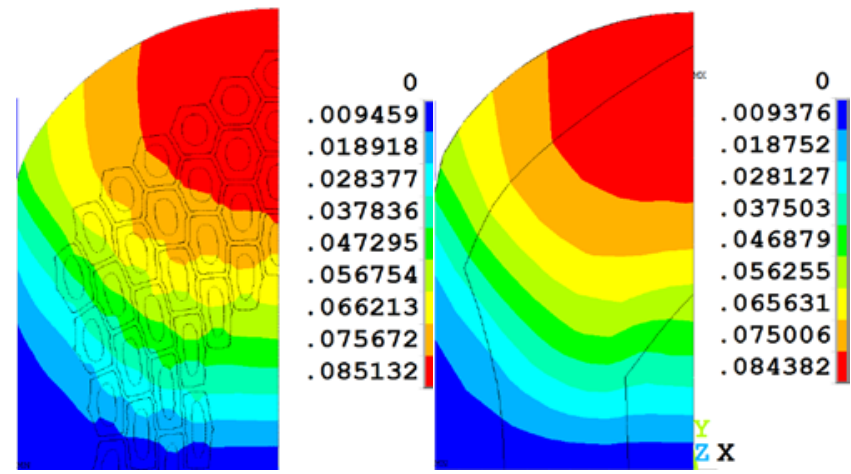

Fig. 2. Y-component of displacement (mm) for detailed (left), and simplified (right) model at $30 \%$ wire deformation.
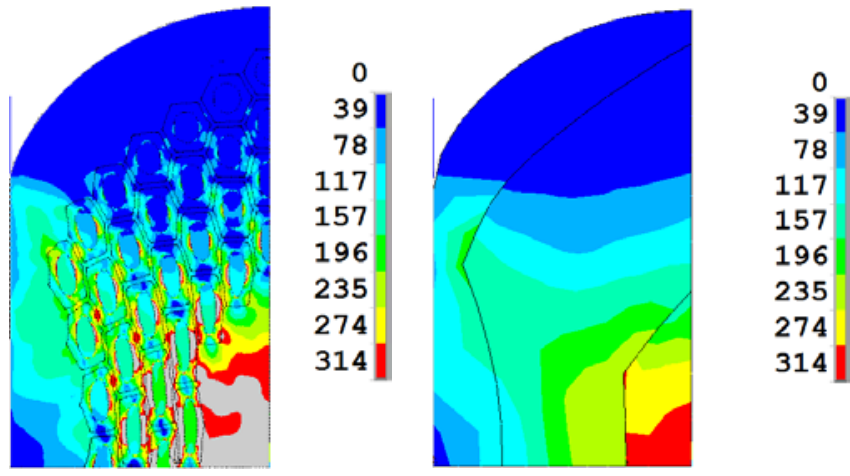

Fig. 3. Plastic work $\left(\mathrm{J} / \mathrm{mm}^{3}\right)$ for detailed (left), and simplified (right) model at $30 \%$ wire deformation.

\section{B. Whole FEM Cable Model and Load Distribution}

The simplified strand model was used to build the whole cable model (see Fig. 4). The undeformed geometry for a cable with odd number of strands can be described by the following equations, which follow from Figs. 4 and 5:

$$
\begin{gathered}
x_{\text {undef }}=2 \bar{x}+(N-1) r^{\prime} \\
y_{\text {undef }}=2 d
\end{gathered}
$$

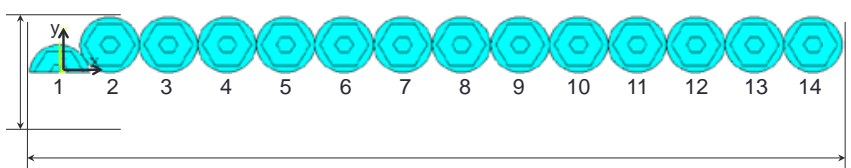

Fig. 4. Whole cable model, undeformed.

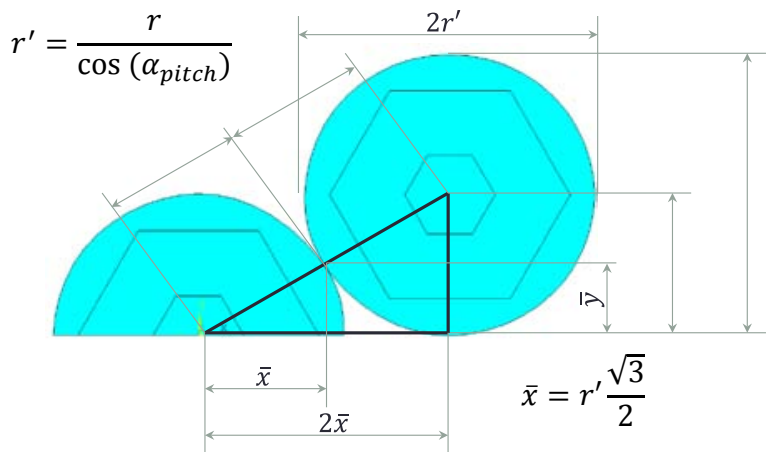

Fig. 5. Undeformed cable geometry.

The cabling procedure presently used at FNAL requires a two stage cable fabrication: first a rectangular cable with narrower width and lower packing factor, and next a keystoned cable with final cross section. The rectangular cables are made using a 42-spool compact cabling machine [14], and a forming fixture made of two vertical rolls with variable gap and two horizontal rolls. The second, keystoning, cabling step is made using a two-roll die with variable gap, and with fixed keystone angle and cable width. For the first rectangular forming stage of the cable, to reproduce the compaction effect of the top and bottom rollers and of the side rollers, vertical and lateral displacement values were imposed, as shown in Fig. 6. Fig. 7 shows the model results (vertical displacement and plastic work) for a lateral displacement applied all on one edge (load case A). Fig. 8 shows the model 
results for a lateral displacement equally distributed on the two edges (load case B).

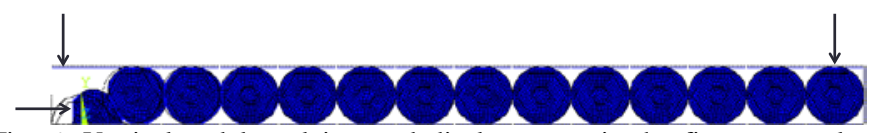

Fig. 6. Vertical and lateral imposed displacements in the first rectangular forming stage.

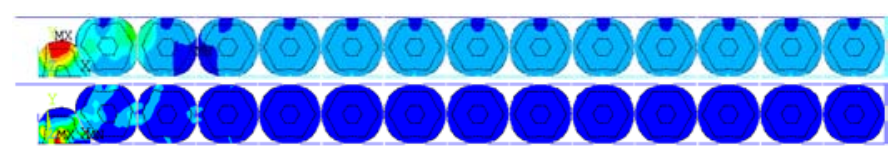

Fig. 7. Y-component of displacement (top) and plastic work (bottom) for cable modeled with load all on one edge.

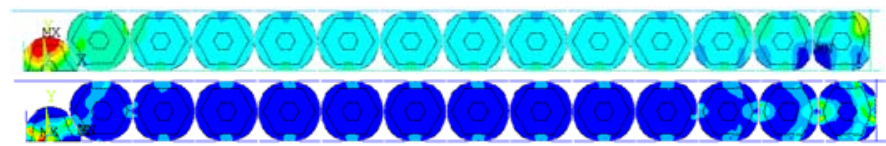

Fig. 8. Y-component of displacement (top) and plastic work (bottom) for cable modeled with load equally distributed on the two edges.

To determine which load distribution best represents cable fabrication, a number of rectangular cables with odd and even number of strands were modeled, and the strands displacements from the model compared with those experimentally measured with a microscope on the actual cable cross sections.

The study was first performed on a cable with 27 (odd) number of strands. Table IV shows the comparison between the average of the longest strand diameter in the straight section of the cable as obtained by the model in the two load cases A and B with that measured on three cable cross sections from strand 4 to 11 (see Fig. 4 for strand location in cable). Load case A provided the least error between model and data. Figs. 9 and 10 show a comparison between model and experimental images in both load case $\mathrm{A}$ and $\mathrm{B}$ for the end strands. In this case too, load case A matches the experimental results better than load case $B$.

TABLE IV LONGEST STRAND DiAMETER AVERAGE

\begin{tabular}{|c|c|c|c|c|c|}
\hline \multirow[b]{2}{*}{ Sample } & \multirow{2}{*}{$\begin{array}{c}\text { Experiment } \\
(\mathrm{mm})\end{array}$} & \multicolumn{4}{|c|}{ "Model } \\
\hline & & $\begin{array}{l}\text { Load Case A } \\
(\mathrm{mm})\end{array}$ & Error \% & $\begin{array}{c}\text { Load Case B } \\
(\mathrm{mm})\end{array}$ & Error \% \\
\hline 1 & 0.726 & & 0.55 & & 1.39 \\
\hline 2 & 0.725 & 0.722 & 0.41 & 0.716 & 1.26 \\
\hline 3 & 0.727 & & 0.69 & & 1.53 \\
\hline
\end{tabular}

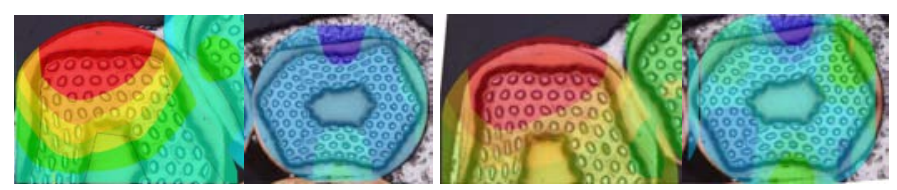

Fig. 9. Left and right edge in load case A (left) and B (right).

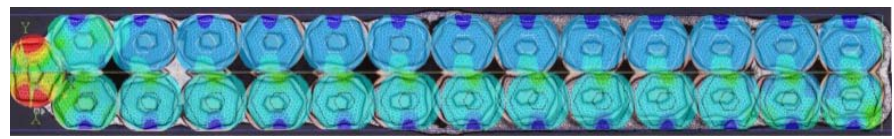

Fig. 10. FEM analysis of whole cable in load case A (top part) compared with load case B (bottom part).

The load case study was performed next for a cable with 40 (even) number of strands. When applying an equal load on both cable edges, symmetry allowed reducing the model to a quarter of the total cable size. Results obtained by these simulations were compared with experimental results of a deformed cable. For a cable with even number of strands, it was found that applying the entire load on one edge (load case A) deformed the cable cross section excessively with respect to the data. Instead, the model predicted very well cable deformation with the load distributed between the two edges. For the latter case, Fig. 11 and Table V show the comparison between model and data for three of the most significant parameters in the cable. The error on the average of the longest strand diameter from strand 3 to 10 in the straight section of the cable is very close to that obtained for the cable with odd number of strands with load case A. It has to be noted that whereas a cable with even number of strands sees a symmetric load (i.e. half of the total load on each edge), its largest undeformed width is larger than in the case of the cable with odd number of strands. The total load on the cable is typically double than in the latter case, therefore producing a similar load on each edge as in the case of the cable with odd number of strands.

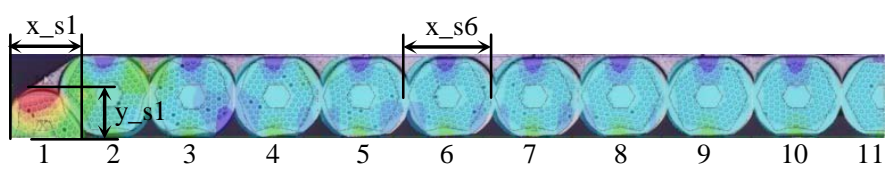

Fig. 11. Deformation comparison between experimental and FEM results.

TABLE V MEASURES COMPARISON

\begin{tabular}{cccc}
\hline \hline Measure & Experiment $(\mathrm{mm})$ & Model $(\mathrm{mm})$ & Error \% \\
\hline X_s1 & 0.613 & 0.613 & -0.05 \\
2*y_s1 & 0.803 & 0.828 & -3.1 \\
Average x_s3,10 & 0.717 & 0.712 & 0.65 \\
\hline \hline
\end{tabular}

The second keystoning stage of cable fabrication [14] was modeled by imposing an additional vertical displacement varying linearly on the strands, as shown in Fig. 12.

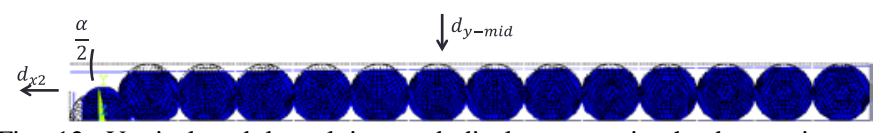

Fig. 12. Vertical and lateral imposed displacements in the keystoning stage.

Fig. 13 shows a comparison between the plastic work generated by the two load steps: as shown in the picture, keystoning increases the average values of plastic work, but does not substantially increase the maximum value, which is primarily determined by the rectangular step of the deformation.

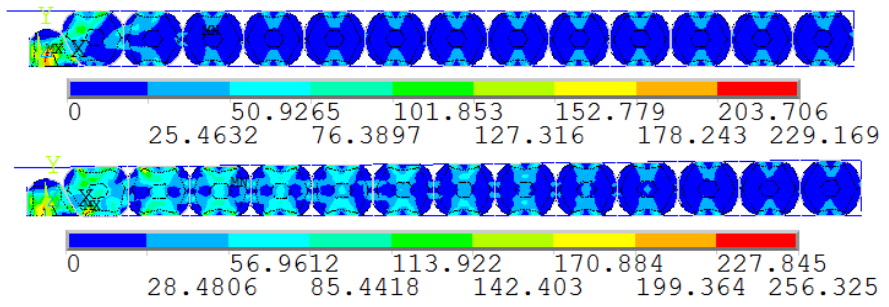

Fig. 13. Plastic work in rectangular (top) and keystoning (bottom) load step. 
The plastic work distribution shown in Fig. 14 identified which are the most critical strands in the cable to be modeled in detail.

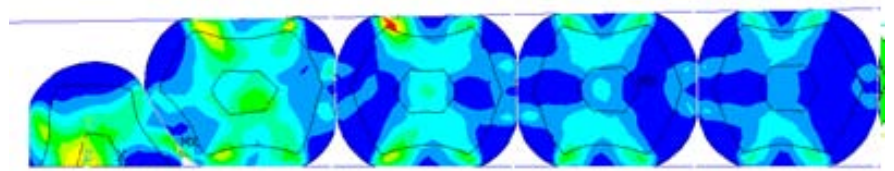

Fig. 14. Detail of plastic work in keystoned cable.

\section{Sensitivity to Number of Strands in Cable}

A study was performed on the effect of the number of strands $N$ in the cable on maximum strand deformation. It was found that for a constant lateral displacement applied onto the cable (case 1 in Fig. 15), the maximum y-component of displacement in the cable did not change for $N$ larger than 7 . Instead, when using the same relative lateral compaction (case 2 in Fig. 15), the maximum y-component in the cable increased linearly.

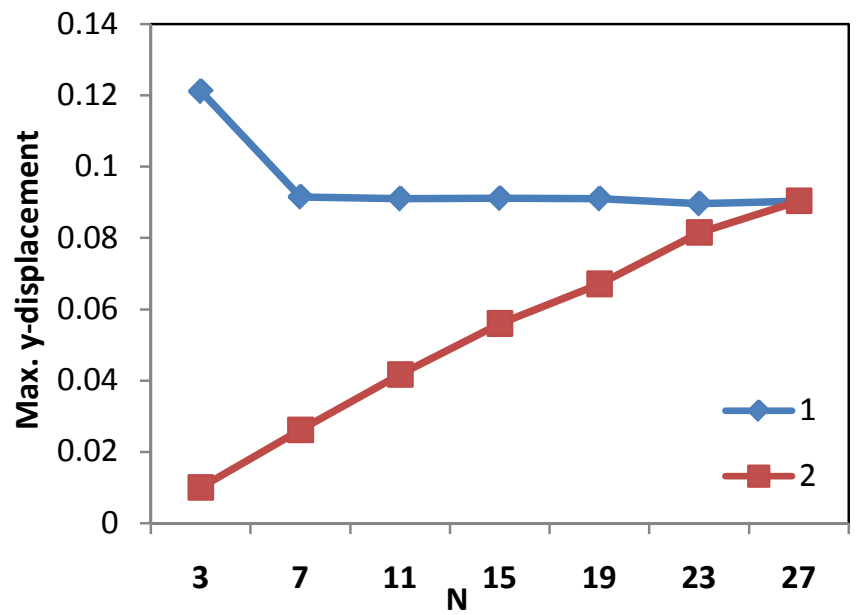

Fig. 15. Maximum y-component of displacement in cable as a function of the number of strands $\mathrm{N}$ in the cable for constant absolute lateral load and for constant relative lateral load.

\section{Detailed Model of Critical Strands in Cable}

In order to identify local strain and critical locations inside the composite strands in the cable, the most critical strands in the cable cross section were modeled in detail by using surface displacements obtained through the simplified cable model (Fig. 16).
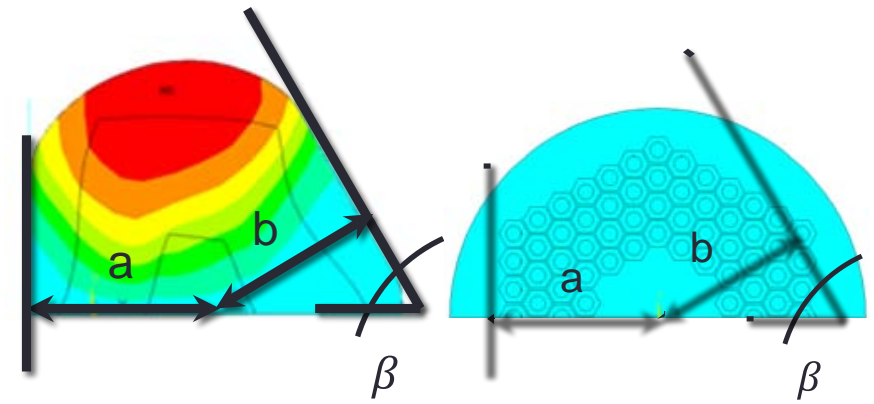

Fig. 16. Detailed modeling of critical strands in cable through surface displacements obtained from cable model.

For both the 27-strand and 40-strand rectangular cables that were modeled, a damage analysis was performed on 6 cable cross sections. Damaged areas that were observed were compared with FEM analysis results. Fig. 17 shows as an example such a comparison for a damaged area found in the 40-strand cable. The damaged areas in the actual cable was associated to areas in the model with principal traction strain in the $\mathrm{Cu}$ channels between the superconducting hexagons in excess of 0.48 , as observed in the single strand analysis in [4]. The same correlation was found in the 27-strand cable between damaged areas and plastic strains.

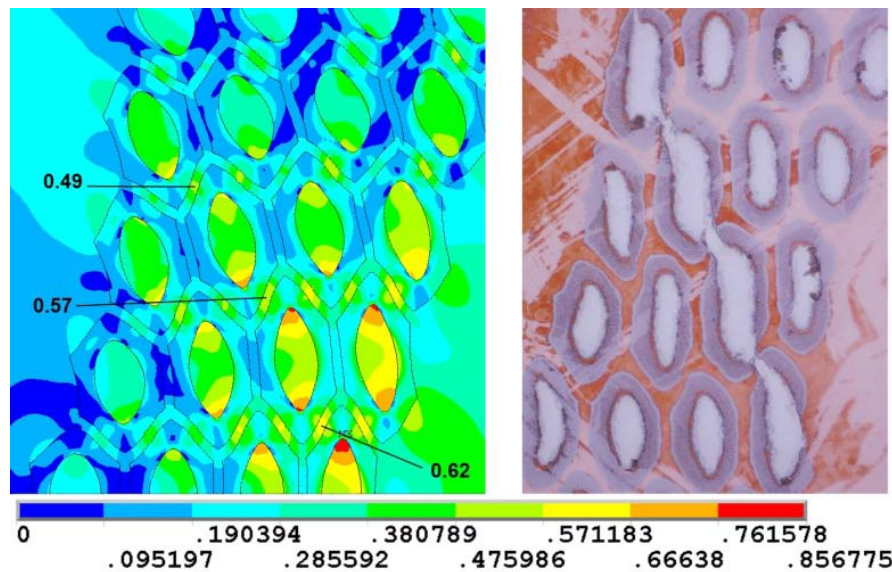

Fig. 17. Plastic strain values in the critical areas where the actual cable appears damaged.

\section{CONCLUSION}

Local strains and critical locations in Rutherford-type NbSn cables were identified by modeling in detail the most critical strands in the cable cross section by using surface displacements obtained through a simplified cable model. The latter had been optimize to obtain the same displacements as in an original detailed strand model [4].

\section{REFERENCES}

[1] S. Farinon et al., "Finite Element Model to Study the Deformations of $\mathrm{Nb}_{3} \mathrm{Sn}$ Wires for the Next European Dipole (NED)”, IEEE Transaction Appl. Sup., V. 17, No. 2, pp. 1136-1139 (2007).

[2] S. Farinon, T. Boutboul, A. Devred, D. Leroy and L. Oberli, "Nb33n Wire Layout Optimization to Reduce Cabling Degradation”, IEEE Transaction Appl. Sup., V. 18, No. 2, pp. 984-988 (2008).

[3] M. Alsharoa'a et al., "Optimization of Brittle Superconducting $\mathrm{Nb}_{3} \mathrm{Sn}$ Strand Designs”, IEEE Transaction Appl. Sup., V. 18, No. 2, pp. 14961499 (2008).

[4] E. Barzi, M. Bossert, and G. Gallo, "A Model to Study Plastic Deformation in $\mathrm{Nb}_{3} \mathrm{Sn}$ Wires”, IEEE Trans. Appl. Sup., V. 21, No. 3, p. 2588 (2011).

[5] H. Bajas, D. Durville, D. Ciazynski, A. Devred, "Numerical Simulation of the Mechanical Behavior of ITER Cable-In Conduit Conductors", IEEE Transaction Appl. Sup., V. 20, No. 3, pp. 1467-1470 (2010).

[6] D. Arbelaez et al., "Cable Deformation Simulation and a Hierarchical

[7] Framework for $\mathrm{Nb}_{3} \mathrm{Sn}$ Rutherford Cables”, EUCAS 09), source: Journal of Physics: Conference Series Volume: 234 Pages: 022002 (11 pp.) DOI: 10.1088/1742-6596/234/2/022002 Published: 012010.

[8] D.P. Boso, M.J. Lefik, B.A. Schrefler, "Thermo-Mechanics of the Hierarchical Structure of ITER Superconducting Cables”, IEEE Trans. on Appl. Sup., vol. 17/2, pp. 1362-1365, 2007.

[9] E. Barzi et al., "Performance of $\mathrm{Nb}_{3} \mathrm{Sn}$ RRP Strands and Cables Based on a 108/127 Stack Design”, IEEE Trans. Appl. Sup., V. 17, No. 2, p. 2718 (2007).

[10] M. B. Field, J. A. Parrell, Y. Zhang, M. Meinesz, and S. Hong, "Internal tin $\mathrm{Nb}_{3} \mathrm{Sn}$ conductors for particle accelerators and fusion applications", Adv. Cryo. Engr., V. 54, pp. 237-243 (2008). 
[11] ANSYS, Inc., Southpointe, 275 Technology Drive. Canonsburg, PA, 15137, U.S.A. Available online: http://www.ansys.com.

[12] Atlas of stress-strain curves, $2^{\text {nd }}$ Ed. The materials information societyASM International, Materials Park, $\mathrm{OH}$.

[13] MatWeb. Material Property Data. Available online: http://www.matweb.com.

[14] N. Andreev et al., "Development of Rutherford-type Cables for High Field Accelerator Magnets at Fermilab”, IEEE Trans. Appl. Sup., V. 17, No. 2, p. 1027 (2007). 